ANN. ZOOTECH.

\author{
I.N.R.A \\ BIBLIOTHEQUE UO 35903 \\ DOMAINE DE CROUELLE \\ 63039 \\ CLERMONT-FD CEDEX 2 \\ $7 / 2 / \mathrm{CO}$
}

$(9$, I960)

\title{
MESURE DE LA PRODUCTION LAITIÈRE DES BREBIS PENDANT LA PÉRIODE D'ALLAITEMENT
}

PAR

\section{G. RICORDEAU, R. BOCCARD, R. DENAMUR}

Avec la collaboration technique de P. Petrequis

Station de Recherches sur l'Élevage et Station de Recherches de l'hysiologie animale, C. N. R. Z., Jouy-en-Joas (S. et O.)

\section{SOMMAIRE}

Après avoir étudié le comportement naturel des agneaux à la mamelle, nous avons cherché à mettre au point une méthode précise et pratique, permettant l'estimation de la production laitière des brebis au cours de l'allaitement, par pesée des agneaux avant et après la tétée.

I. L'intervalle de tétée des agneaux Texel et Préalpes est en moyenne de 1'ordre de I h 30 ; il n'est jamais supérieur à 3 heures.

Le comportement des agneaux à la mamelle est peu différent en ce qui concerne les simples et les bessons.

La fréquence des tétées varie au cours de la journée et en fonction du poids des agneaux, mais le facteur le plus important est l'âge des agneaux.

2. Nous avons enregistré la production laitière de I4 brebis Préalpes pendant deux périodes de 48 heures, à la suite de doubles pesées effectuées toutes les deux heures et toutes les trois heures.

Du point de vue production laitière, il n'y a pas de différence significative entre deux jours successifs de contrôle ou deux demi-journées de contrôle.

L'estimation de la production laitière en 24 heures est bonne quand les pesées portent sur une période de r2 heures, avec des intervalles de tétée de 2 ou 3 heures. Dans les deux cas, 1'écart-type des différences relatives entre production réelle et production estimée est de l'ordre de 6 à 7 p. Ioo.

La mesure de la production laitière des brebis au cours de l'allaitement nécessite la mise au point d'une méthode de contrôle particulière.

Une des techniques le plus souvent utilisée est la mesure indirecte de la production des mères par pesée des agneaux avant et après la tétée. 
La mise en ouvre de cette technique implique un certain nombre de conditions : le nombre de pesées au cours d'un contrôle journalier doit tenir compte de la précision des pesées, de la répétabilité des mesures et leur fréquence doit être aussi voisine que possible du rythme naturel des tétées. La périodicité des contrôles journaliers doit, elle-même, tenir compte de la précision de ces contrôles et de la variabilité de la production.

Notre travail comprend deux parties essentielles :

A. - Fréquence naturelle des tétées.

B. - Étude d'une méthode de détermination de la production laitière pendant l'allaitement, par pesées des agneaux avant et après la tétée.

\section{A. - FREQUENCE NATURELLE DES TETEES}

La fréquence des tétées dépend à la fois du comportement de la mère et de celui de l'agneau. WALLACE (I948) a souligné le rôle important de la brebis. Au début de 1'allaitement les jeunes peuvent téter aussi souvent qu'ils le désirent; avec l'avancement de la lactation, la brebis tolère moins bien les tétées et en écourte la durée. BARNICOAT C. R., Logan A. G., Grant A. I. (I949) ont observé que les agneaux de 4 et 6 semaines tétaient environ 6 fois par jour. Munro (1956) a procédé à une analyse plus complète, portant à la fois sur le rythme et la durée des tétées. Des contrôles, effectués sur deux brebis avec simples et deux brebis avec bessons, lui permettent de conclure que les tentatives de tétées, fructueuses ou non, sont en $\mathrm{I} 6$ heures de $\mathrm{I} 5$ à 45 pour les bessons et de 9 à 25 pourr les simples. Quand l'âge des agneaux augmente, la fréquence et la durée des tétées diminuent.

\section{MATÉRIEL ET METHODES.}

Nos observations du comportement alimentaire des agneaux eurent lieu en bergerie sur deux lots de brebis et d'agneaux.

Io Dans une première série, un lot de race Préalpes, constitué de 13 brebis et 16 agneaux sensiblement de même âge, fut observé chaque semaine pendant 24 heures les deux premières semaines, puis pendant I2 heures (de 8 à 20 heures) jusqu'à la sixième semaine.

$2^{\circ}$ La seconde série porta sur un lot de race Texel, constitué de I4 brebis et de $2 \mathrm{I}$ agneaux. Les observations furent menées pendant I2 heures (de 8 à 20 heures) à 4 stades différents de la lactation. Deux observateurs se sont attachés à n'enregistrer que les tétées jugées effectives. 


\section{RÉSULTATS}

\section{a. - RôLE DES AGNEAUX.}

Io Le nombre d'agneanx par brebis.

A partir de nos observations, nous pouvons dresser les tableaux I et II et le graphique I.

\section{TABLEAU I}

$1^{\text {er }}$ cas : Bessons

\begin{tabular}{|c|c|c|}
\hline & Texel & Préalpes \\
\hline 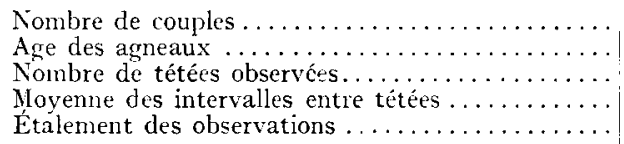 & 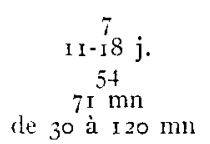 & 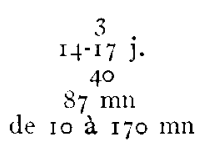 \\
\hline
\end{tabular}

TABlEAu II

2 e cas: Simples

\begin{tabular}{|c|c|c|}
\hline & Texel & Préalpes \\
\hline $\begin{array}{l}\text { Nombre d'agneaux } \ldots \ldots \ldots \ldots \ldots \ldots \ldots \ldots \ldots \ldots \ldots \\
\text { Age des agneaux } \ldots \ldots \ldots \ldots \ldots \ldots \ldots \ldots \ldots \\
\text { Nombre de tétées observées } \ldots \ldots \ldots \ldots \ldots \ldots \ldots \\
\text { Moyenne des intervalles entre tétées } \ldots \ldots \ldots \ldots \ldots \\
\text { Étalement des observations } \ldots \ldots \ldots \ldots \ldots \ldots \ldots\end{array}$ & $\begin{array}{c}\text { moins de } \frac{9}{3} \text { semaines } \\
64 \\
\text { de } 30 \text { à } \mathrm{m} \text { I } 80 \mathrm{~mm}\end{array}$ & $\begin{array}{c}8 \\
\text { I }+ \text { - } 77 \mathrm{j} \\
\text { I } \mathrm{I} S \\
87 \mathrm{~mm} \\
\text { de } 10 \text { a } \mathrm{I} 70 \mathrm{~mm}\end{array}$ \\
\hline
\end{tabular}

- a) Agneaux bessons : dans le cas des agneaux Texel, tous les intervalles entre tétées sont inférieurs à 2 heures. Pour les agneaux Préalpes, $80 \mathrm{p}$. Ioo des tétées ont lieu dans l'intervalle de 2 heures.

- b) Agneaux simples : l'intervalle entre tétées est semblable à celui observé pour les bessons. Cependant, pour les agneaux Texel, Io p. roo des intervalles sont supérieurs à 2 heures. Dans le cas des Préalpes, les tétées sont encore moins régulières, mais $80 \mathrm{p}$. Ioo d'entre elles se répètent dans les 2 heures.

La fréquence des tétées des agneaux simples et des bessons n'est donc pas différente. L'hypothèse de WALLACE selon laquelle les brebis bessonnières produisent plus de lait, car elles sont sollicitées plus souvent par leurs agneaux, ne peut done être confirmée.

Notons, d'autre part, que les agneaux de race Texel, de même âge que les agneaux Préalpes, ont une fréquence de tétée un peu plus grande. 

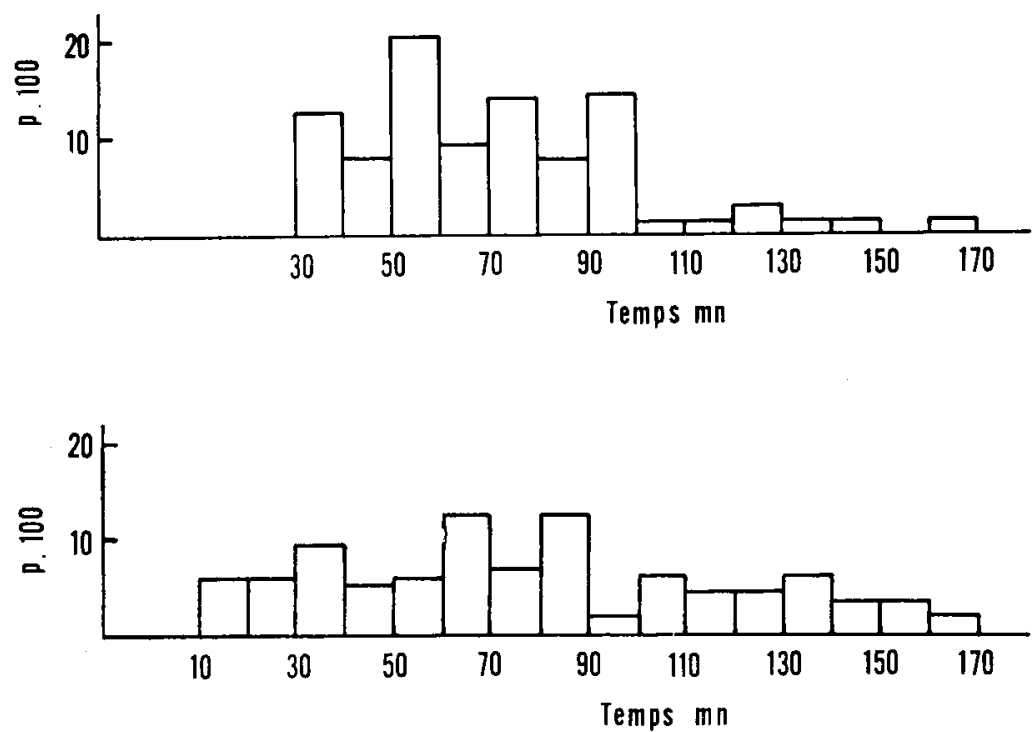

Graphique I. - Intervalle de tétées pour des agneaux Texel et Préalpes de 2 à 3 semaines. Haut - 9 mères avec simples, 'Texel (64 obs.). Bas - 8 mires avec simples, Préalpes (I 15 obs.)

Ordonnée : p. Ioo du nombre d'observations ; abscisse : intervalle de tétíes.
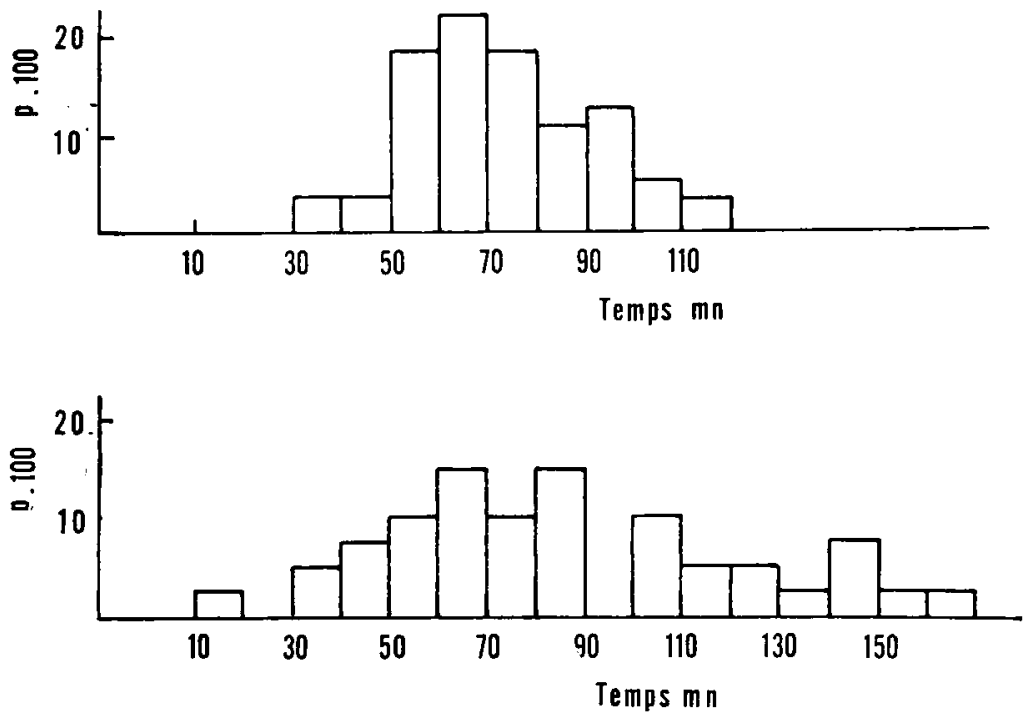

Graphique I bis. - Intervalle de tétées pour des agneaux Texel et Préalpes de 2 à 3 semaines. Haut - 7 mères avec bessons, Texel (54 obs.). Bas - 3 mères avec bessons, Préalpes (40 obs.).

Ordonnée : p. Ioo du nombre d'observations. Abscisse : intervalle des tétées. 


$$
2^{\circ} \text { Age des agneaux. }
$$

Ein utilisant les données concernant toutes les tétées des agneaux Préalpes, obtenues entre $8 \mathrm{~h}$ le matin et $20 \mathrm{~h}$ le soir, nous pouvons dresser le tableau III.

'TABLEAU III

\begin{tabular}{|c|c|c|c|c|c|c|}
\hline \multirow[b]{2}{*}{$\begin{array}{l}\text { No d'ordre } \\
\text { des contrôles }\end{array}$} & \multicolumn{2}{|c|}{8 agneaux simples } & \multicolumn{2}{|c|}{3 couples de bessons } & \multicolumn{2}{|c|}{ Moyenne des i a brebis } \\
\hline & $\begin{array}{l}\text { Age moyen } \\
\text { au contrôle }\end{array}$ & $\begin{array}{l}\text { Intervalle } \\
\text { entre tétée } \\
\text { (moyenne) }\end{array}$ & $\begin{array}{l}\text { Age moyen } \\
\text { au contrôle }\end{array}$ & $\begin{array}{l}\text { Intervalle } \\
\text { entre tétée } \\
\text { (moyenne) }\end{array}$ & $\begin{array}{l}\text { Age moyen } \\
\text { au contrôle }\end{array}$ & $\begin{array}{l}\text { Intervalle } \\
\text { entre tétée } \\
\text { (moyemne) }\end{array}$ \\
\hline & (j) & $(m n)$ & (j) & $(\mathrm{mn})$ & (j) & $(\mathrm{mn})$ \\
\hline I $\ldots \ldots \ldots \ldots$ & 6 & $88 *$ & 8 & 87 & 7 & 88 \\
\hline $2 \ldots \ldots \ldots \ldots$ & I4 & 9 I & I 6 & $9^{8}$ & I 5 & 95 \\
\hline $3 \ldots \ldots \ldots \ldots$ & $2 \mathrm{I}$ & 91 & 23 & 107 & 22 & 95 \\
\hline $4 \ldots \ldots \ldots \ldots$ & $3^{6}$ & 97 & 38 & 108 & 37 & 10.3 \\
\hline $5 \ldots \ldots \ldots \ldots$ & 44 & 171 & $4^{6}$ & 207 & 45 & $18 \mathrm{I}$ \\
\hline
\end{tabular}

* A ce contrôle, 7 agneaux simples seulement ont été considérés, l'un d'entre eux étant malade.

Les différences observées entre simples et bessons ne peuvent être considérées comme significatives, étant donné le petit nombre d'agneaux observés et la grande variabilité des intervalles entre les tétées.

Le seul fait notable est la brutale augmentation de l'intervalle entre tétées, à partir de la $6^{\mathrm{e}}$ semaine. Cette diminution de la fréquence des tétées peut être expliquée par le comportement des mères qui supportent très mal les tétées à partir de ce moment et qui, souvent, se dérobent. Quant aux agneaux, ils consomment à cet âge une quantité appréciable d'aliment concentré. Ce début d'indépendance alimentaire peut également expliquer partiellement la diminution de fréquence des tétées.

Notons que, par ailleurs, la durée des tétées, qui peut atteindre plusieurs minutes les deux premières semaines, se réduit à quelques dizaines de secondes après 5 semaines.

\section{$3^{\circ}$ Poids des agneaux à la naissance.}

I,e poids des agneaux à la naissance est un facteur de vigueur (THoısoN, I953) et de capacité à téter rapidement et souvent. Nous avons voulu vérifier si ce facteur conservait son influence avec l'augmentation de l'âge des agneaux.

De nos observations, nous pouvons extraire les éléments du tableau IV, indiquant les intervalles (ell minutes) entre tétées pour des agneaux simples, du même âge, mais de poids à la naissance différent. 
TABLEAU IV

\begin{tabular}{|c|c|c|c|c|c|c|}
\hline & \multirow{2}{*}{$\begin{array}{l}\text { Poids moyen } \\
\text { à la } \\
\text { naissance }\end{array}$} & \multicolumn{5}{|c|}{ Intervalles entre tétées } \\
\hline & & $\begin{array}{c}\mathrm{I}^{\mathrm{re}} \\
\text { semaine }\end{array}$ & $\begin{array}{c}z^{e m e} \\
\text { semaine }\end{array}$ & $\begin{array}{l}3^{\mathrm{eme}} \\
\text { semaine }\end{array}$ & $\begin{array}{l}5^{\mathrm{eme}} \\
\text { semaine }\end{array}$ & $\begin{array}{l}\text { 6eme } \\
\text { semaine }\end{array}$ \\
\hline $\begin{array}{c}\text { Groupe } I: \\
5 \text { agneaux } \ldots \ldots \ldots\end{array}$ & $3,8 \mathrm{~kg}$ & $95 \mathrm{mn} *$ & $90 \mathrm{mn}$ & $92 \mathrm{mn}$ & $102 \mathrm{~mm}$ & $162 \mathrm{~mm}$ \\
\hline $\begin{array}{c}\text { Groupe } I I: \\
3 \text { agneaux ........ }\end{array}$ & $5,+\mathrm{kg}$ & $79 \mathrm{mn}$ & $89 \mathrm{mn}$ & $89 \mathrm{~mm}$ & $90 \mathrm{mn}$ & $188 \mathrm{~mm}$ \\
\hline
\end{tabular}

* Lors de ce contrôle, un des agneaux malade a été exclu.

Les intervalles entre tétées ne semblent pas conditionnés par le poids à la naissance, bien que vers l'âge de 7 jours les agneaux lourds tètent légèrement plus souvent. S'il existe des différences importantes dans le comportement selon le poids à la naissance, elles ne doivent se manifester que dans les tous premiers jours de vie. L'âge ou le stade de lactation apparaissent plus importants que le poids des agneaux dans la détermination de la fréquence des tétées.

\section{$4^{\circ}$ Variation du comportement diurne et nocturne.}

Dans les deux premières séries d'observations, nous avons enregistré le comportement des agneaux Préalpes pendant 24 heures. Au cours de la nuit, la bergerie était très faiblement éclairée et la lecture du numéro des agneaux était effectuée à l'aide d'une torche électrique. Nous ne pensons pas que ce faible éclairement ait modifié sensiblement les habitudes nocturnes des agneaux.

Les résultats obtenus entre 6 heures et $\mathrm{I} 8$ heures (jour) et $2 \mathrm{I}$ heures 30 et 6 heures (nuit) nous permettent de dresser le tableau $\mathrm{V}$.

TABLEAU V

\begin{tabular}{|c|c|c|c|c|c|c|}
\hline & \multicolumn{6}{|c|}{ Intervalles entre tétées } \\
\hline & \multicolumn{3}{|c|}{ dans le cas des simpies } & \multicolumn{3}{|c|}{ dans le cas des bessons } \\
\hline & $\begin{array}{l}\text { Nombre } \\
\text { de brebis }\end{array}$ & Jour & Nuit & $\begin{array}{l}\text { Nombre } \\
\text { de brebis }\end{array}$ & Jour & Nuit \\
\hline $\begin{array}{l}\text { 1er controle : } \\
\text { (âge moyen : } 7 \text { jours) .. } \\
\text { 2e contrôle: }\end{array}$ & 7 & $88 \mathrm{mn}$ & $105 \mathrm{mn}$ & 3 & $87 \mathrm{~mm}$ & $\mathrm{I} 03 \mathrm{~mm}$ \\
\hline (âge moyen : 15 jours).. & 8 & $90 \mathrm{mn}$ & I I I mn & 3 & $97 \mathrm{mn}$ & $92 \mathrm{~mm}$ \\
\hline
\end{tabular}

Dans l'ensemble, la fréquence des tétées est plus faible au cours de la nuit; seuls les bessons ne manifestent aucune différence lors de la $2^{\mathrm{e}}$ semaine. 


\section{b. - QUalités MATERNELLES DE LA BREBIS}

A l'exception de la production laitière, les facteurs propres aux brebis ne sont pas faciles à mesurer objectivement.

Nos observations sur le comportement du groupe d'animaux Préalpes furent suivis chaque semaine par un contrôle de la production laitière des brebis, par pesées des agneaux avant et après la tétée. Les pesées se déroulèrent toutes les deux heures sur une période de I2 heures.

Il ne nous fut pas possible de mettre en évidence des relations entre la quantité de lait consommée au cours de ce contrôle et la fréquence naturelle des tétées observée la veille (contrôles $3,4,5$ ) ou au maximum l'avant-veille (contrôles I et 2 ).

Nous avons déjà $v \mathfrak{u}$, à propos du comportement des simples et des bessons, que leur fréquence de tétées était sensiblement la même. On ne peut donc concevoir que la production laitière supérieure des bessonnières soit imputable à la plus grande fréquence de sollicitation des mamelles par les detux agneaux. La tétée simultanée des deux agneaux pourrait agir sur la production laitière :

- en induisant des influx nerveux (fréquence, intensité etc...) plus efficaces sur la décharge hormonale posthypophysaire et antéhypophysaire,

- par évacuation plus rapide du lait de la glande mammaire assurant une vidange plus complète de celle-ci, pendant la brève action de 1'ocytocine sur le myoépithélium. Ceci pourrait expliquer pourquoi la traite simultanée, mais relativement lente, d'un quartier pendant que l'agneau vidange rapidement l'autre ne permet d'obtenir que 6o-75 p. Ioo du lait tété (Constantinescu et Gondos, I958).

\section{B. - ETUDE D'UNE METHODE DE DETERMINATION DE LA PRODUCTION LAITIÉRE PENDANT L'ALLAITEMENT, PAR PESEE DES AGNEAUX AVANT ET APRES LA TETEE}

Les premières tentatives concernant l'estimation de la production laitière des brebis, par pesée des agneaux avant et après la tétée, ont été faites par Fuller et Kleinheinz (Ig04) sur I4 brebis. Par la suite, de nombreuses expériences, utilisant cette méthode, ont été poursuivies par différents chercheurs. Le tableau VI résume les techniques utilisées.

A partir des résultats d'un certain nombre de pesées d'agneaux avant et après la tétée, nous avons étudié pour nos besoins expérimentaux une méthode de contrôle de la production laitière pendant la période d'allaitement. 


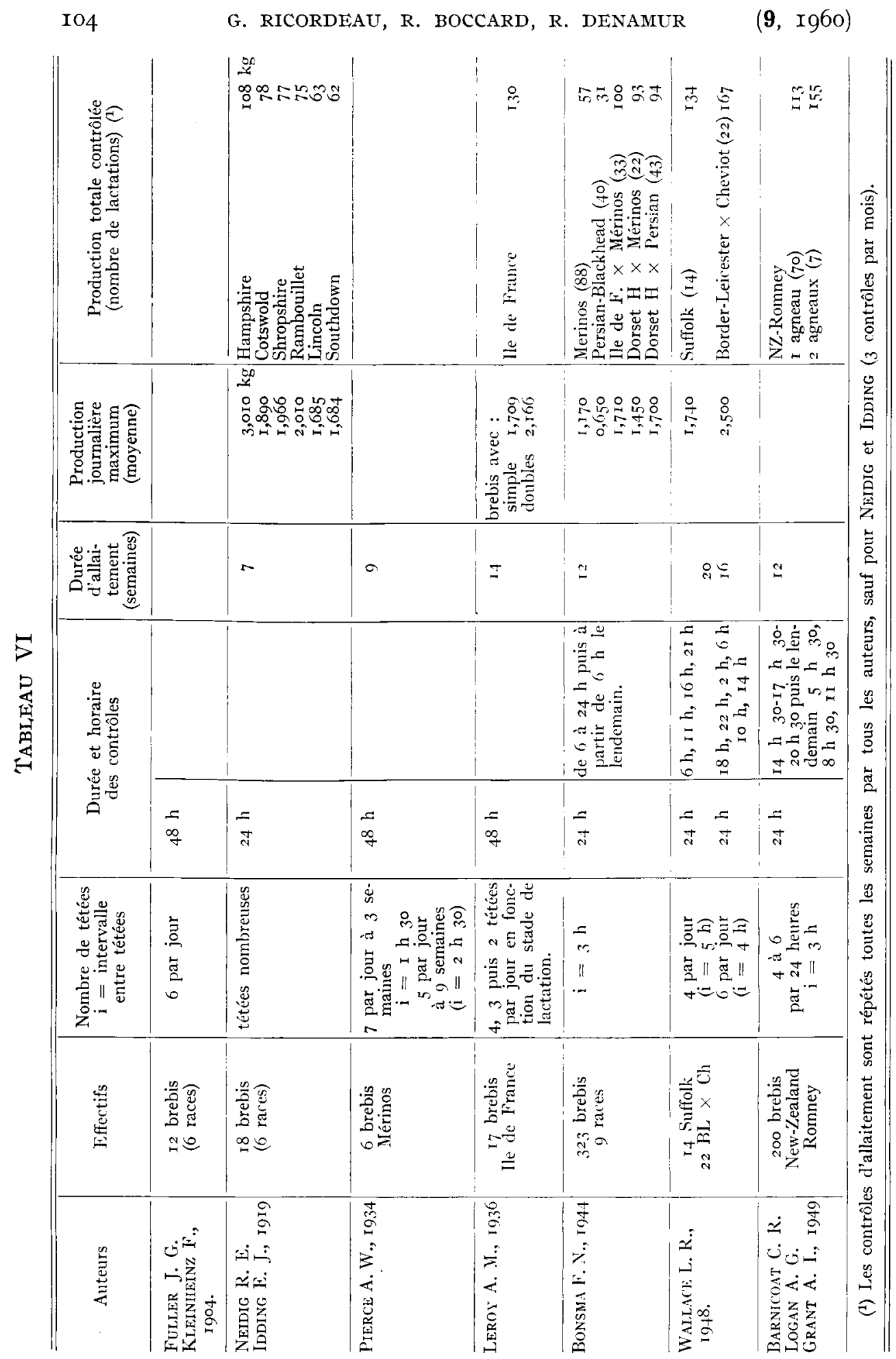


(9, I960)

PRODUCTION IAITIËE DES BREBIS

IO5

\begin{tabular}{|c|c|c|c|c|c|c|c|c|c|c|}
\hline 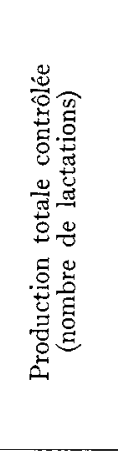 & & $\begin{array}{l}\text { so } \\
8 \\
8 \\
\approx \\
i\end{array}$ & 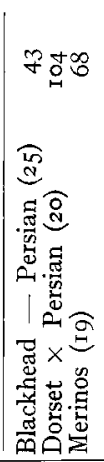 & 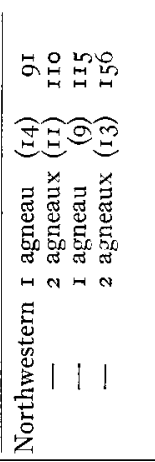 & 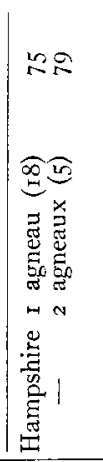 & 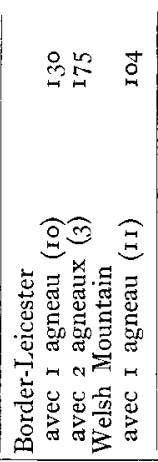 & 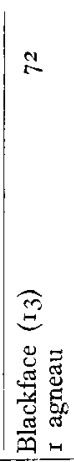 & 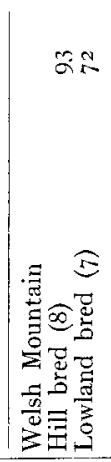 & & 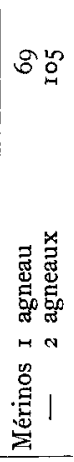 \\
\hline 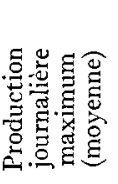 & 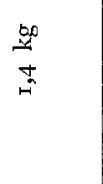 & & & 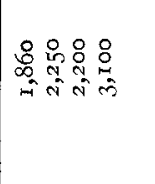 & 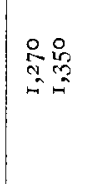 & 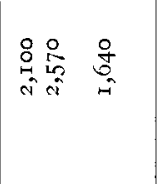 & \begin{tabular}{l}
0 \\
\multirow{2}{*}{} \\
\hdashline \\
\hdashline
\end{tabular} & 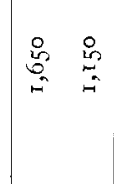 & & 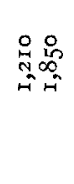 \\
\hline 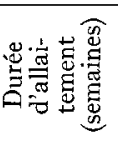 & 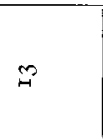 & $\approx$ & $\approx$ & $a$ & $\Xi$ & $\because$ & 0 & 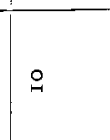 & 0 & 웅 \\
\hline 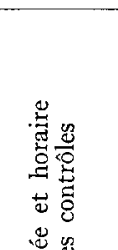 & 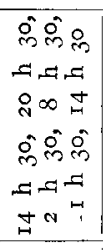 & 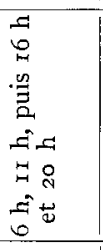 & & 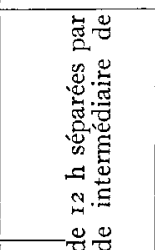 & 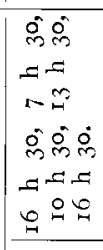 & 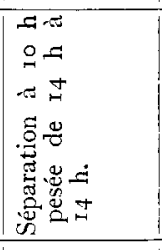 & & & \multirow{2}{*}{ 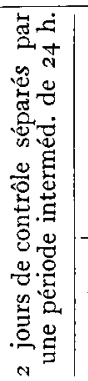 } & \multirow{2}{*}{$\begin{array}{c} \\
\\
\\
\\
0\end{array}$} \\
\hline 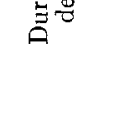 & & & & 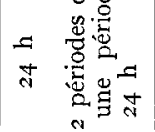 & $\frac{7}{d}$ & $\underset{\pi}{A}$ & $\stackrel{5}{a}$ & $\underset{\mathrm{a}}{\Rightarrow}$ & & \\
\hline 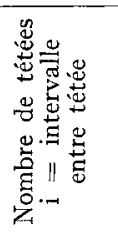 & 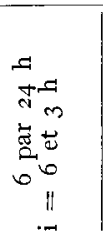 & 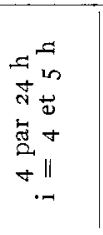 & 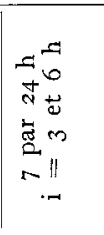 & 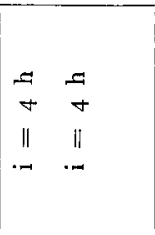 & 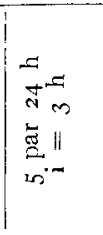 & $\begin{array}{l}\because \\
. \\
.1\end{array}$ & 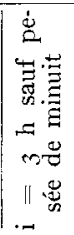 & $\begin{array}{l}n \\
z \\
7\end{array}$ & a & in \\
\hline 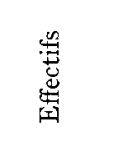 & 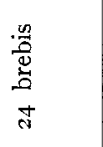 & 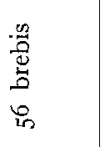 & & 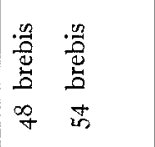 & $\begin{array}{l}\frac{m}{2} \\
\frac{2}{2} \\
0 \\
a\end{array}$ & 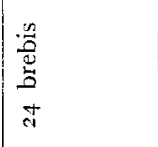 & 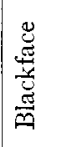 & 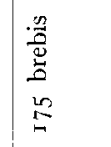 & 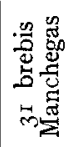 & $\begin{array}{l}\text { 总 } \\
\text { 龸 } \\
\text { लै }\end{array}$ \\
\hline 戀 & 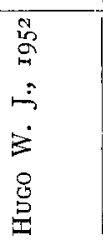 & 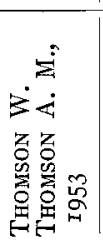 & 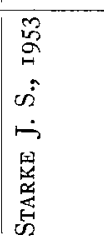 & 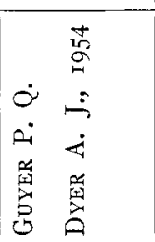 & 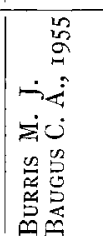 & 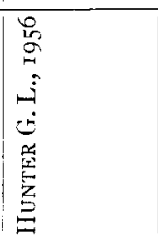 & 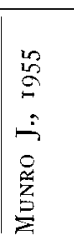 & 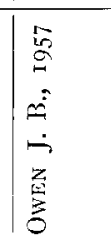 & 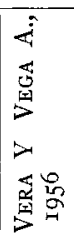 & 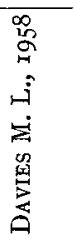 \\
\hline
\end{tabular}




\section{MATÉRIEL E'T MÉTHODES}

I a précision des contrôles est, dans une grande mesure, liée à la précision de la balance utilisée pour la pesée des agneaux. Les quantités de lait ingérées mesurées par différence entre le poids enregistré avant et après la tétée, sont de l'ordre de quelques centaines de grammes. I1 est donc nécessaire d'opérer avec une balance sensible, sur une échelle de poids assez grande (o à $40 \mathrm{~kg}$ ), pour diminuer les erreurs de mesure. Nous avons adopté une balance d'une portée de $40 \mathrm{~kg}$ (graduations par Io $\mathrm{g}$, freinage rapide) avec un indicateur électro-optique.

Il importe que la contention de l'agneau soit réalisée an mieux, afin d'obtenir une bonne stabilisation durant la lecture. Les mouvements de l'agneau sont réduits au minimum lorsque celui-ci est retourné, aussi avons-nous construit un appareil de contention voisin de celui utilisé par GUYER et DYER (I954). Cet appareil à la forme d'un V. Pour qu'il puisse servir à des sujets de poids très différents, les parois latérales sont plus resserrées à une extrémité qu'à 1'autre.

La plus grande partie de nos observations a été réalisée en bergerie. Les mères étaient logées en cases; une porte séparait ces cases d'un parc mitoyen où l'on groupait les agneaux après la tétée.

$\mathrm{Au}$ moment du contrôle, les agneaux étaient pesés toujours dans le même ordre. On n'a jamais fait téter plus de deux agneaux à la fois. Cette façon de procéder permettait une surveillance plus efficace et évitait les erreurs dues aux mictions et aux défécations. L'intervalle de temps qui s'écoule entre les deux pesées, avant et après la tétée, est alors aussi bref que possible, mais cette technique limite le nombre d'agneaux à I 5 ou 20 au maximum pour chaque opération.

Pour les erreurs accidentelles de poids des agneaux au cours des pesées, nous avions constaté au cours d'une expérience préalable sur des Texel, que les erreurs pouvant résulter de la miction ou de la défécation étaient approximativement les suivantes :

\begin{tabular}{|c|c|c|}
\hline Age de l'agneau & Urine & Fèces \\
\hline o à 3 semaines & $20 \mathrm{gr}$ & $5 g$ \\
\hline$\ldots \ldots \ldots \ldots \ldots$ & $30 \mathrm{~g}$ & IO \\
\hline 6 à 9 semaines $\ldots$ & 508 & I5 g \\
\hline
\end{tabular}

En faisant téter un ou deux agneaux à la fois, on arrive facilement à éliminer ces causes d'erreurs. Si toutefois un agneau urine, il est facile de corriger le résultat de sa pesée, mais ce procédé doit être l'exception.

Afin d'éviter de perturber les mères en les séparant de leurs agneaux au cours de la journée de contrôle, nous avions pensé utiliser la technique du protège mamelle, utilisé par OWEN (r956). L'emploi de ce protège 


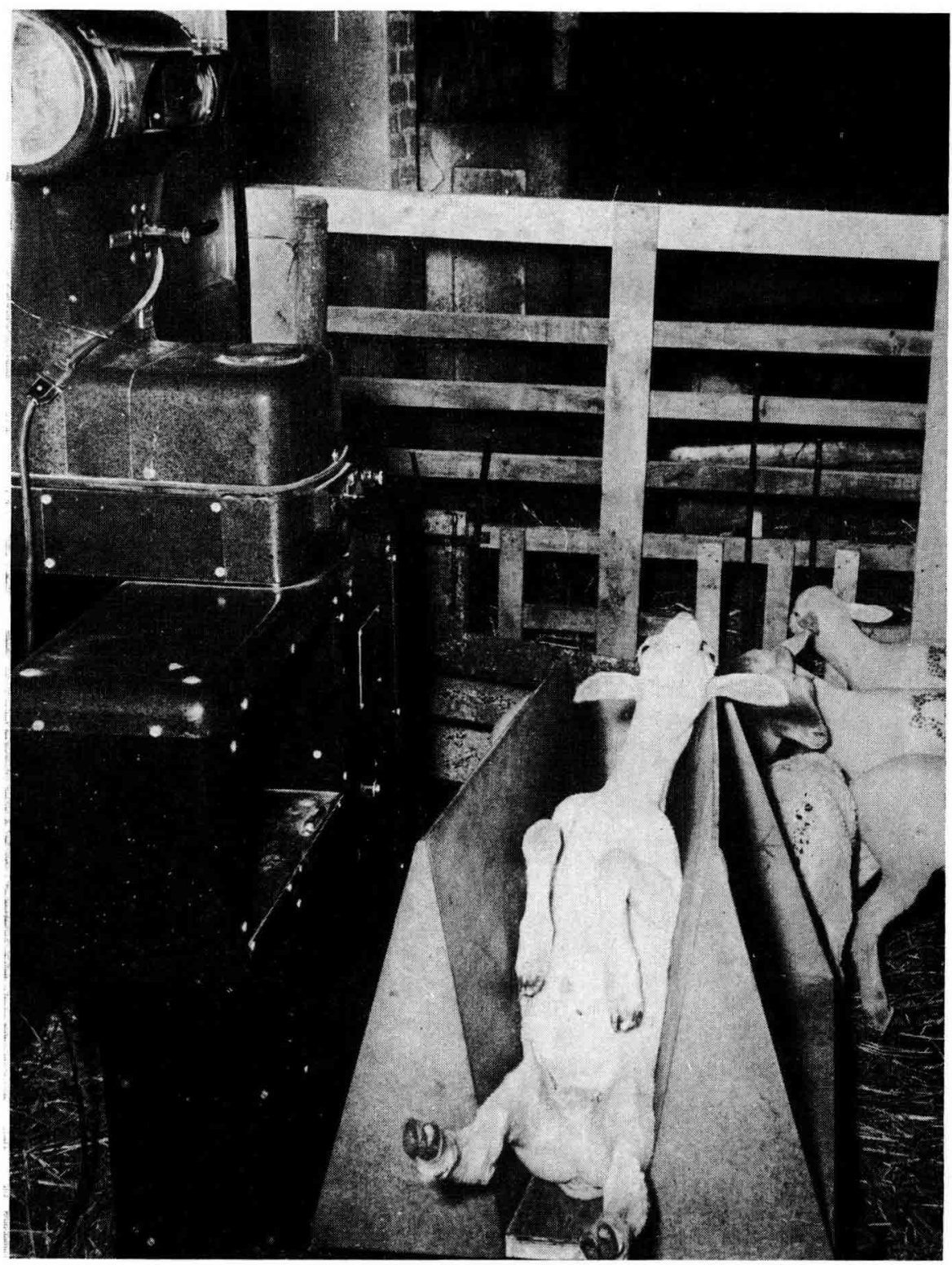


mamelle permet aux agneaux de rester près de leur mère, mais présente toutefois un certain nombre d'inconvénients non négligeables:

- difficultés d'adaptation (types de mamelles différents),

- pose difficile,

et, finalement, perturbation des brebis.

\section{TECHNIQUE IUU CONTROLE}

Nous pouvons constater, d'après le tableau VI, que les techniques utilisées par les différents auteurs sont assez variables. Il est bien évident, d'ailleurs, qu'il existe de nombreuses combinaisons correspondant aux différents besoins et possibilités.

L'étude précédente nous a appris :

Io Que 1'intervalle moyen de tétée était de 1'ordre de $87 \mathrm{mn}$,

$2^{\circ}$ Que $80 \mathrm{p}$. roo des tétées avaient lieu dans un intervalle de 2 heures.

$3^{\circ}$ Que l'intervalle maximum entre deux tétées était de 3 heures.

Compte tenu de ces observations, nous avons expérimenté deux méthodes de contrôle :

- l'une basée sur des pesées à $2_{\mathbf{t}}^{-}$heures d'intervalle : Période A,

- 1'autre basée sur des pesées à 3 heures d'intervalle: Période $B$. Voici le schéma expérimental utilisé :

\begin{tabular}{|c|c|c|c|}
\hline $\begin{array}{l}\text { Date } \\
\text { du contróle: }\end{array}$ & $\begin{array}{l}\text { Numéro } \\
\text { du contróle } \\
\text {-- }\end{array}$ & $\begin{array}{l}\text { Intervalle } \\
\text { entre tétée }\end{array}$ & $\begin{array}{l}\text { Nombre de tétées } \\
\text { en } 24 \text { heures }\end{array}$ \\
\hline II-3-1957....... & I & 2 heures & I 2 Période $A$ \\
\hline I $2-3-1957 \ldots \ldots \ldots$ & 2 & 2 heures & I 2 \\
\hline $19-3-1957 \ldots \ldots \ldots$ & 3 & 3 heures & 8 Période $\mathrm{B}$ \\
\hline $20-3-1957 \ldots \ldots \ldots$ & 4 & 3 heures & 8 \\
\hline
\end{tabular}

- Nombre d'animaux :

I4 antenaises avec I agneau

- Age des agneaux :

2 à 3 semaines (moyenne I 8 jours aux contrôles $\mathrm{I}$ et 2 )

3 à 4 semaines (moyenne 26 jours aux contrôles 3 et 4.)

Période A :

Ire séparation des agneaux à 5 h 30 le I I-3-I957

Pesée zéro à 7 h 30

Ire pesée effective à 9 h 30 .

Période B :

${ }^{\text {re }}$ séparation des agneaux à 5 h le I9-3-I957

Pesée zéro à $8 \mathrm{~h}$

I $^{\text {re }}$ pesée effective à II h.

Les quantités de lait consommées à la première pesée n'ont pas été retenues, l'intervalle de tétée correspondant pouvant être très variable. 


\section{RESULTATS}

\section{I. - Variations de la quantité de lait consommée par les agneaux au cours d'une période de 48 heures.}

Nous avons enregistré la production laitière des brebis pendant $4^{8}$ heures, à la suite de doubles pesées effectuées toutes les deux heures et toutes les trois heures; le tableau VII et le graphique II indiquent les résultats obtenus.

L'examen des résultats fait apparaître des variations qui peuvent avoir des origines différentes:

- variations systématiques, dues aux heures de pesées ou d'affouragement,

--- variations aléatoires.

Il importe d'analyser les résultats en vue de mettre en évidence 1a nature et l'importance de ces variations. A partir des données de deux périodes $\mathrm{A}$ et $\mathrm{B}$, nous avons effectué 1'analyse de variance des quantités de lait consommées par les agneaux à chaque tétée. Les résultats sont inscrits dans le tableau VIII.

- Les différences entre brebis sont significatives dans les deux cas. En 24 heures, dans les périodes $\mathrm{A}$ et $\mathrm{B}$ respectivement, la meilleure brebis a donné I 730 et I $825 \mathrm{~g}$ de lait, contre I r 20 et I $035 \mathrm{~g}$ à la plus faible laitière. Ia moyenne des $\mathrm{I}_{4}$ brebis était de I $370 \mathrm{~g}$ dans la période $\mathrm{A}$ et de I $425 \mathrm{~g}$ dans la période $\mathrm{B}$.

- Entre les différentes mesures, il existe des différences significatives. Toutefois, si ces différences dépassent à peine le seuil de signification dans la période $A$, elles sont très significatives dans la période $B$. Quelle est la nature de cette variation? Esst-ce une variation systématique due à l'influence des heures de pesée, ou une variation aléatoire et incontrôlable? Il y a donc lieu de calculer l'interaction entre jour de contrôle et heures de tétée. Dans la période A, cette interaction est statistiquement peu significative; compte tenu du faible nombre de degrés de liberté, c'est-à-dire de l'importance des erreurs d'échantillonnage, on ne peut tirer aucune conclusion rigoureuse de ce résultat. Par contre, cette interaction est significative à partir des données de la période $\mathrm{B}$. I1 semble donc exister une certaine variation systématique de la quantité de lait consommée par les agneaux au cours d'une expérience de 24 heures, les tétées du matin étant légèrement défavorables, alors que celles de l'après-midi et de la soirée sont supérieures à la moyenne journalière.

Nous avons cherché à expliquer l'origine possible de ces variations horaires. Au cours de nombreux contrôles, effectués ultérieurement, nous avons pu faire deux constatations importantes :

- La variabilité des mesures est beaucoup plus importante le jour que la nuit ; 


\section{TABLEAU VII}

Quantités de lait consommées

par les agneaux au cours d'une période de 48 heures.

\begin{tabular}{|c|c|c|c|c|c|}
\hline \multicolumn{3}{|c|}{ Période A } & \multicolumn{3}{|c|}{ Période B } \\
\hline $\begin{array}{l}\text { Heures } \\
\text { de tétées }\end{array}$ & ${ }^{\text {re journée }}$ & $2^{\mathrm{e}}$ journée & $\begin{array}{l}\text { Heures } \\
\text { de tétées }\end{array}$ & I $^{\text {re }}$ journée & $2^{\mathrm{e}}$ journée \\
\hline $9 h 30 \ldots \ldots \ldots$ & $\mathrm{I} 02 \mathrm{~g}$ & $129 \mathrm{gr}$ & I I $\mathrm{h}$. & $145 \mathrm{~g}$ & II $6 \mathrm{~g}$ \\
\hline I I h $30 \ldots \ldots \ldots$ & $97 \mathrm{~g}$ & $88 \mathrm{~g}$ & I $h$ & $\mathrm{I} 87 \mathrm{~g}$ & I. $85 \mathrm{~g}$ \\
\hline r $3 \mathrm{~h} 30 \ldots \ldots \ldots$ & $\mathrm{I} 52 \mathrm{~g}$ & $127 \mathrm{~g}$ & $14 \pi$ & $107 \mathrm{~g}$ & $100 \mathrm{~g}$ \\
\hline I 5 h $30 \ldots \ldots \ldots$ & $90 \mathrm{~g}$ & $122 \mathrm{~g}$ & I $7 \mathrm{~h}$ & $\mathrm{x} 69 \mathrm{~g}$ & $176 \mathrm{~g}$ \\
\hline $\begin{array}{llll}\text { I } 7 & h & 30 & \ldots\end{array}$ & $\begin{array}{ll}\text { I } 19 & g \\
\text { I } 6 & g\end{array}$ & $\begin{array}{l}\mathrm{I} 22 \mathrm{~g} \\
13^{6} \mathrm{~g}\end{array}$ & $20 \mathrm{~h}$ & I99 $\mathrm{g}$ & $216 \mathrm{gr}$ \\
\hline $2 \mathrm{I} h 30 \ldots \ldots \ldots$ & $10+8$ & 950 & $23 \mathrm{~h}$ & I $85 \mathrm{~g}$ & $208 \mathrm{~g}$ \\
\hline 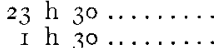 & $\begin{array}{l}\mathrm{x} \text { I } 4 \mathrm{~g} \\
\mathrm{I} 8 \mathrm{~g}\end{array}$ & $\begin{array}{l}\text { I } 28 \% \\
\text { I } 198\end{array}$ & $2 h \ldots \ldots \ldots$ & $210 \mathrm{~g}$ & $22 \mathrm{Ig}$ \\
\hline $3 \mathrm{~h} 30 \ldots \ldots \ldots$ & $124 \mathrm{~g}$ & 1228 & $5 \mathrm{~h} \ldots$ & $\mathrm{I} 74 \mathrm{gr}$ & $15^{2} \mathrm{~g}$ \\
\hline $\begin{array}{c}5 \\
5 \\
7\end{array}$ & $\begin{array}{l}\text { II } 6 \mathrm{~g} \\
\text { IOI } \mathrm{g}\end{array}$ & $\begin{array}{r}88 \mathrm{~g} \\
120 \mathrm{~g}\end{array}$ & $8 h \ldots \ldots$. & I68 g & I33 g \\
\hline Moyenne ..... & I. $5 \mathrm{~g}$ & I $6 \mathrm{~g}$ & Moyenne. & $180 \mathrm{~g}$ & $176 \mathrm{~g}$ \\
\hline
\end{tabular}

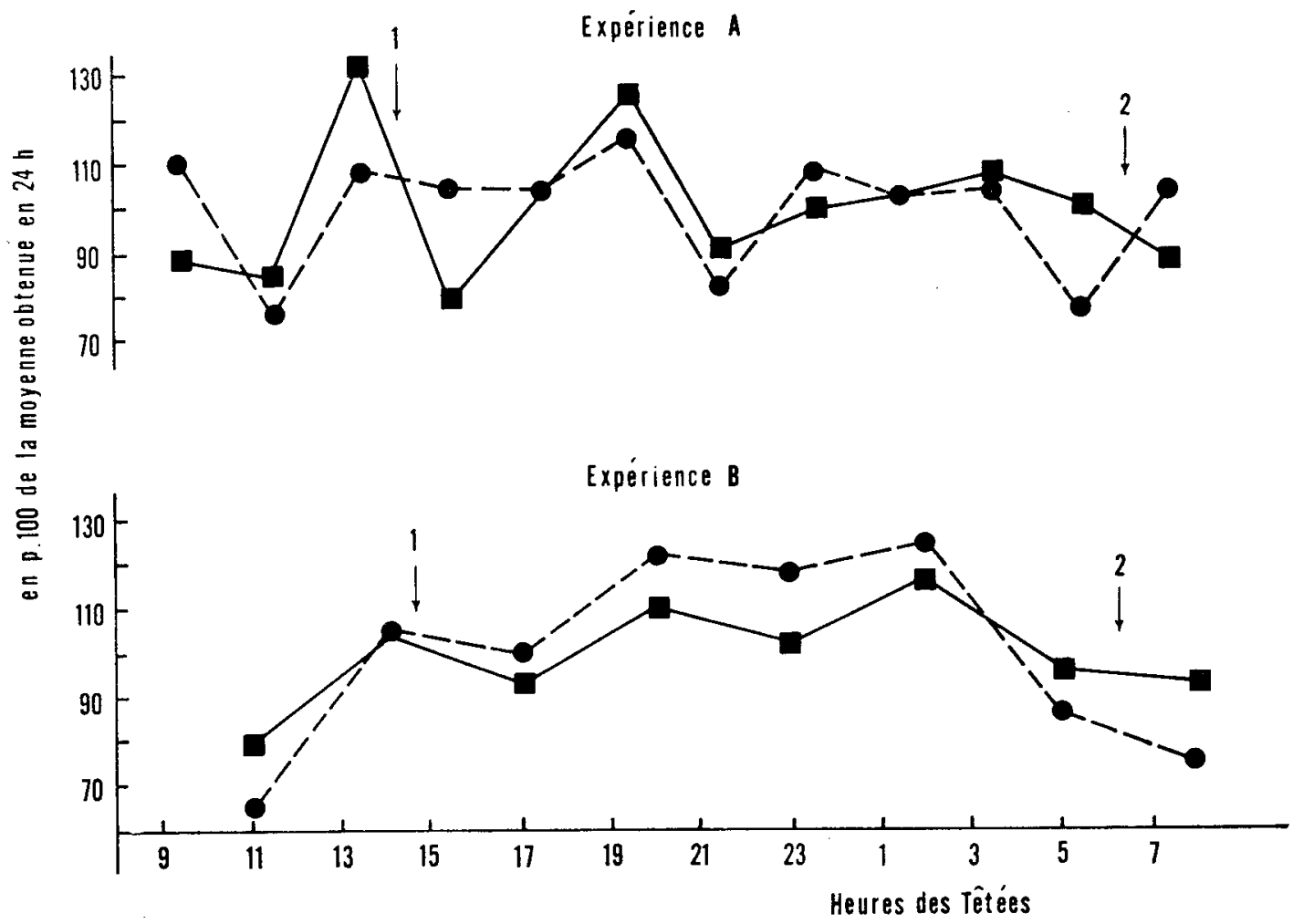

Graphique II. - Variations de la quantité de lait consommée par les agneaux à chaque tétée, au cours d'une période de 48 heures.

I - betteraves et fourrage

2 - concentré

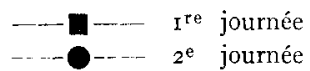


TABLEAU VIII

Analyse de la variance des quantités de lait consommées par les agneaux à chaque tétée au cours d'une période de 48 heures.

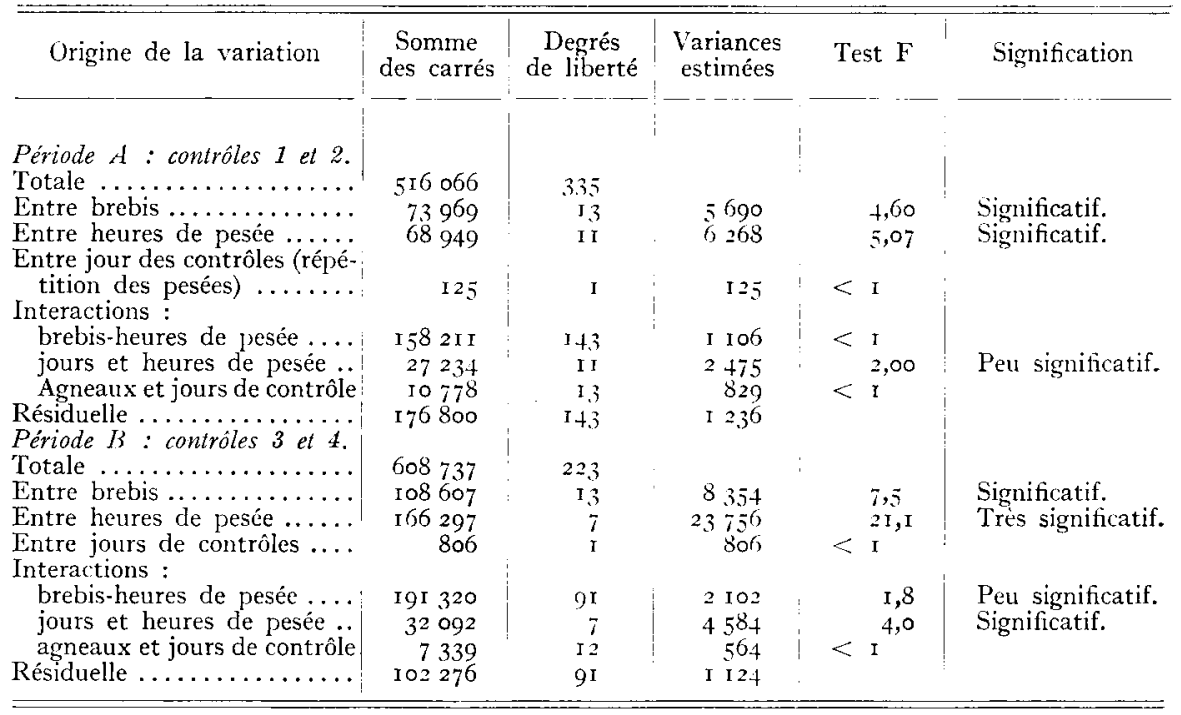

- il ne semble pas y avoir une influence systématique de l'heure de pesée, mais simplement des perturbations dues à un ensemble de facteurs plus ou moins constants d'un jour à l'autre, comme l'heure d'affouragement et l'appétit des agneaux.

En effet, après chaque affouragement, les brebis se laissent téter moins facilement et la tétée est moins complète. Au contrôle suivant les résultats sont supérieurs. Dans la soirée, et surtout la nuit, la bergerie est souvent plus calme, les animaux se reposent et les tétées sont beaucoup plus régulières et complètes. Pour réduire ou éliminer les variations horaires, systématiques ou non, qui n'apparaissent pas totalement négligeables, il semble donc nécessaire d'étaler les pesées sur une période suffisamment longue. Le début de cette période doit être situé le plus tôt possible matin. En effet, par le calcul nous avons établi que la période de 22 heures, la plus représentative de la journée, s'étendait de 5 heures 30 à $I 7$ heures 30 .

- Entre les résultats obtenus au cours de deux périodes consécutives de 24 heures, il n'existe pas de différence significative. Il est donc possible de faire porter les pesées sur une seule journée.

\section{2. - Nombre de pesées à retenir lors d'un contrôle journalier.}

Connaissant les variations de la quantité de lait consommé par les agneaux au cours d'une période de 24 heures, on doit essayer d'effectuer 
les pesées sur une fraction de journée. STARKe (I953) et OWEn (I956), notamment, ont signalé la possibilité de limiter à une demi-journée la période de contrôle. Chez la truie, SAL,MON-LEGAGNEUR (I956) a montré qu'on potvait encore réduire cette période, à condition toutefois de suivre assez étroitement le rythme naturel de tétée des porcelets.

Pour résoudre cette question, nous avons comparé la production réellement obtenue en 24 heures, à la production calculée sur la base des $n$ premières mesures. La différence ainsi obtenue entre la production réelle et la production estimée ou calculée indique l'erreur commise.

Aux erreurs d'échantillonnage près, ces écarts doivent se distribuer selon une loi normale, de moyenne nulle et d'écart-type donné $\sigma_{e}$ (tableau IX).

A partir de la distribution des écarts, le problème consiste donc à tester leur moyenne, à vérifier leur normalité et à estimer leur dispersion.

\section{$\mathrm{I}^{0}$ Signification des moyennes.}

La signification des moyennes a été effectuée grâce au test de Student-Fisher. Le tableau IX (colonnes 3 et 4 ) indique les résultats. Ia moyenne des écarts n'est pas significativement différente de zéro, sauf dans le cas de pesées à 3 heures d'intervalle. Nous retrouvons là l'influence du moment de pesée : influence qui se manifeste uniquement dans le cas de pesées toutes les trois heures. Nous déduisons, également, qu'il n'y a pas de différence significative entre les productions obtenues au cours de chaque demi-journée de contrôle, ou encore entre les périodes diurnes et nocturnes.

\section{$2^{\circ}$ Distribution des écarts : dispersion et normalité.}

Pour chacune des expériences $A$ et $B$, nous disposons de deux séries d'échantillons et de I4 observations. Peut-on supposer que les deux échantillons correspondant à chaque estimation appartiennent au même ensemble, de moyenne nulle et de variance $\sigma_{e}^{2}$. Si les deux échantillons ne sont pas significativement différents, il est done possible de grouper l'ensemble des observations et d'en déduire une valeur approchée de la variance des écarts.

La comparaison des variances par l'application du test de FisherSnédécor ne traduit aucune différence très significative entre les variances. On peut donc estimer que les distributions ont même dispersion. D'autre part, il n'apparaît aucune différence significative entre les moyennes des écarts, sauf pour les deux dernières estimations de l'expérience $B$.

Nous avons supposé jusqu'à maintenant que les ensembles dont proviennent les échantillons sont distribués normalement. Ėn groupant 
$(9$, I960)

PRODUCTION LAITIÈ̀RE DES BREBIS

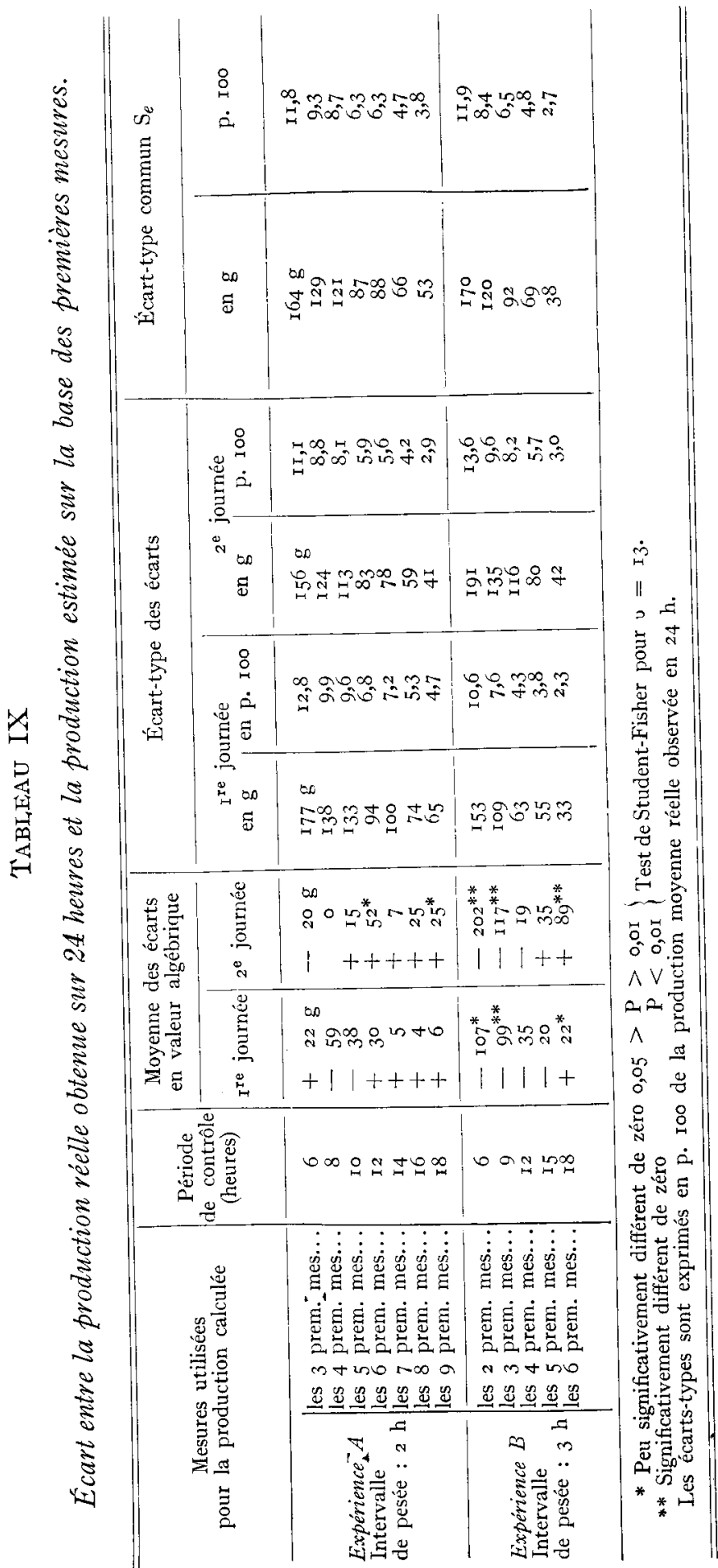

sunale de Zootechnie. - II, rg6o. 
les deux échantillons correspondant à chaque estimation, on peut tester la normalité de la distribution des écarts par la méthode de la droite de HENRY. Malgré le petit nombre d'observations, les graphiques traduisent des distributions approximativement normales.

Dans ces conditions, les 28 observations correspondant à chaque estimation, peuvent être considérées comme appartenant à une même population, de variance commune $s_{e}^{2}$, dont l'espérance mathématique $\left(\sigma_{e}^{2}\right)$ est la variance de l'ensemble des écarts pour une population considérée comme infinie.

Les résultats concernant la variance commune des écarts sont inscrits dans les deux dernières colonnes du tableau IX et sur le graphique III.

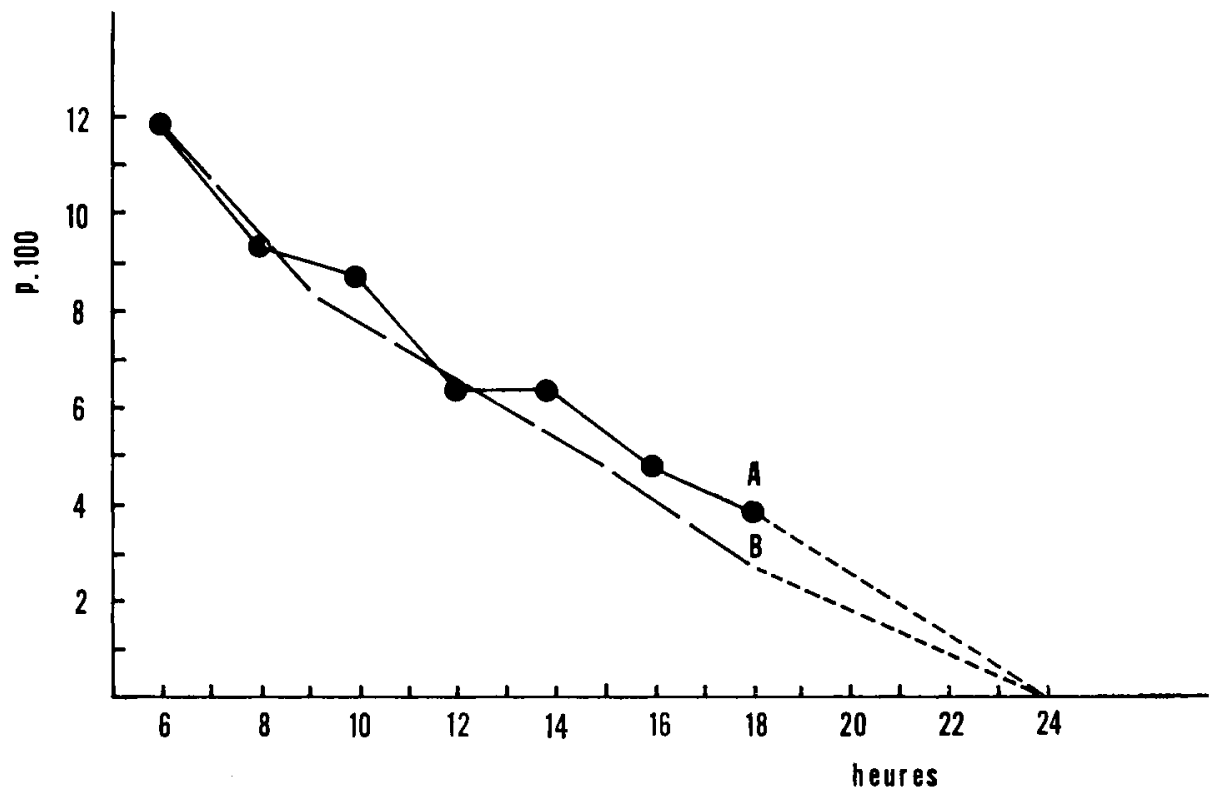

Graphique III. - - Variations de l'écart-type des écarts entre production réelle et production calculée en fonction de la période de contrôle et du nombre de mesures.

Ordonnée : écart-type des écarts en p. ioo de la production moyenne réelle.

Abscisse : période sur laquelle portent les contrôles

Il reste maintenant à préciser l'intervalle de confiance de cette variance commune.

$3^{0}$ Signification de la variance des écarts.

Limitons-nous au calcul de l'intervalle de confiance de la variance des écarts dans le cas du contrôle portant uniquement sur une période de 12 heures.

Pour chaque expérience, nous disposons donc de 28 observations, 
c'est-à-dire d'un petit échantillon. Les lois de probabilité des variables aléatoires $s^{2}$ et $s$ ne peuvent être considérées comme normales. Pour estimer la variance, utilisons le test de $\chi^{2}$ applicable à la variable réduite tel que :

$$
\chi^{2}=\frac{\Sigma(x-\bar{x})^{2}}{\sigma^{2}}
$$

- expérience $A$ :

I2 heures de contrôle - soit 6 tétées à 3 heures d'intervalle

$$
\Sigma(x-\bar{x})^{2}=205.409 \quad \text { pour } \quad \nu=27
$$

- expérience $B$ :

I 2 heures de contrôle - soit 4 tétées à 3 heures d'intervalle

$$
\Sigma(x-\bar{x})^{2}=227.666 \quad \text { pour } \quad \nu=27 .
$$

En se rapportant à une table détailiée de $\chi^{2}$ on voit qu'il y a une probabilité de 0,025 que $\chi^{2}$ soit compris entre 0 et 14,6 et une probabilité identique qu'il soit supérieur à 43,2. Il y a donc une probabilité 0,95 pour que :

$$
{ }_{15}, 6<\chi^{2}=\frac{\Sigma(x-\bar{x})^{2}}{\sigma^{2}}<43,2
$$

\begin{tabular}{|c|c|c|}
\hline \multirow{2}{*}{ Expérience } & \multicolumn{2}{|c|}{ Intervalle de confiance à 0,95 pour $\sigma_{e}$} \\
\hline & $\begin{array}{l}\text { en p. Ioo de la production } \\
\text { moyenne réclle }\end{array}$ & en $g$ \\
\hline $\begin{array}{l}\mathrm{A} \ldots \ldots \ldots \ldots \ldots \ldots \ldots \\
\mathrm{B} \ldots \ldots \ldots \ldots \ldots\end{array}$ & $\begin{array}{l}4,9<\sigma_{e}<8,5 \\
5, \mathrm{I}<\sigma_{e}<8,8\end{array}$ & $\begin{array}{l}69<\sigma_{e}<\text { I } 19 \\
73<\sigma_{e}<\text { I } 25\end{array}$ \\
\hline
\end{tabular}

c'est-à-dire que :

$$
\frac{\Sigma(x-\bar{x})^{2}}{43,2}<\sigma^{2}<\frac{\Sigma(x-\bar{x})^{2}}{\mathrm{I} 4,6}
$$

Les résultats sont les suivants :

La comparaison des erreurs enregistrées au cours des périodes de référence semble indiquer que la précision de l'estimation dépend beaucoup plus de la durée de la période de mesure que du nombre de pesées. Il y aurait donc intérêt à espacer les pesées. Toutefois, dans le cas de pesée à 3 heures d'intervalle, on constate qu'il faut au moins 4 contrôles pour que la différence moyenne entre la production calculée et la production réelle ne soit pas significativement différente de zéro.

En conclusion

La période de contrôle doit être de I2 heures au moins.

Les estimateurs A ( 6 pesées toutes les 2 heures) et B (4 pesées toutes les 3 heures) sont convergents et ont une variance de même ordre de grandeur. 
L'estimateur A est plus rapidement sans distorsion, il est donc le plus efficace.

\section{3. - Influence de l'augmentation de l'intervalle de tétée sur la quantité de lait consommée par les agneaux à intervalles de 4 heures.}

Nous avons vu qu'en choisissant un intervalle de tétée de 2 ou 3 heures (voisin du rythme naturel des agneaux), sur une période de longue durée ( 48 heures), on n'enregistre pas de diminution sensible de la production laitière. Au contraire, certains auteurs adoptant un intervalle de tétée prolongé constatent une chute de production au fur et à mesure du prolongement du contrôle, et ceci quelle que soit 1'heure de la journée à laquelle débutent les contrôles. HunTer (I956)observe ce phénomène sur des brebis séparées de leurs agneaux toutes les 4 heures. Il met en cause la fatigue de la brebis. Nous pensons plutôt que cet interva1le s'éloigne trop du rythme normal des agneaux.

$L_{\text {a }}$ fréquence des tétées a-t-elle une influence sur la quantité de lait consommée? Munro et Inkson (I957) ont comparé les intervalles de I heure et 4 heures sans trouver de différence significative.

En I $95^{8}$, nous avons procédé à une expérience complémentaire portant sur $I_{5}$ brebis allaitant un senl agneau et réparties au hasard en deux lots.

Au cours des premières 24 heures, les 7 brebis du lot I ont été contrôlées toutes les 2 heures; les 8 brebis du lot II, toutes les 4 heures. I+e jour suivant, les agneaux sont restés en liberté avec leur mère. Au cours du troisième jour, l'intervalle de tétée a été inversé.

Les résultats sont les suivants :

\section{Lait consommé en 24 heures}

\begin{tabular}{|c|c|c|}
\hline & ${ }^{\text {er }}$ jour & $3^{\mathrm{e}}$ jour \\
\hline 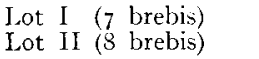 & $\begin{array}{l}\text { I } 328 \mathrm{~g}\left(\begin{array}{ll}2 & \mathrm{~h}\end{array}\right) \\
\text { I } 522 \mathrm{~g}(4 \mathrm{~h})\end{array}$ & $\begin{array}{l}\text { I } 202 \mathrm{~g}\left(\begin{array}{ll}4 & \mathrm{~h}\end{array}\right) \\
\text { I } 555 \mathrm{~g}(2 \mathrm{~h})\end{array}$ \\
\hline Hoyenne & I $457 \mathrm{~g}$ & I $390 \mathrm{~g}$ \\
\hline $\begin{array}{l}\text { Moyenne } 2 \mathrm{~h} \text { : } \\
\text { Moyenne } 4 \mathrm{~h}:\end{array}$ & $\begin{array}{l}\text { I } 474 \\
\text { I } 373\end{array}$ & \\
\hline Différence moyenne : & 10 & \\
\hline
\end{tabular}

Pour évaluer ces différences nous avons effectué l'analyse de variance dont les résultats sont résumés ci-dessous :

\begin{tabular}{|c|c|c|c|c|}
\hline Origine de la variance & Sommes des carrés & (d. l) & Variance & F \\
\hline 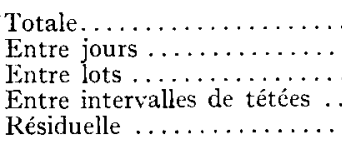 & $\begin{array}{r}2830667 \\
33333 \\
456720 \\
77 \text { Or } 3 \\
2263601\end{array}$ & $\begin{array}{r}20 \\
I \\
I \\
I \\
26\end{array}$ & $\begin{array}{r}33 \quad 333 \\
456 \quad 720 \\
77 \text { or } 3 \\
87 \text { O6I }\end{array}$ & $\begin{array}{l}<\text { I N.S. } \\
\sum_{5,2} \text { I N.S. S. }\end{array}$ \\
\hline
\end{tabular}


Comme on le voit, seule la différence entre les lots est significative. Si la différence entre jours de contrôle est faible ( $67 \mathrm{~g}$ en moyenne), celle relative à l'intervalle de tétée est plus sensible (Ior g en moyenne) bien que statistiquement non significative.

A partir de ces résultats, nous avons voulu vérifier l'importance des erreurs dues au nombre de pesées au cours d'un contrôle journalier.

Si les contrôles avaient été effectués sur les premières I2 heures, c'est-à-dire à partir de 6 pesées dans le cas de tétées toutes les 2 heures, à partir de 3 pesées dans le cas de tétées toutes les 4 heures, l'écart type des écarts entre la production réelle et la production estimée aurait été respectivement de 6,8 p. Ioo (I03 g) et 8,7 p. Ioo (I20 g).

Fin ce qui concerne les tétées à 2 heures d'intervalle, les écarts sont du même ordre de grandeur que ceux enregistrés au cours de l'expérience initiale.

En choisissant un intervalle de tétée de 4 heures, non seulement l'estimation de la quantité de lait consonmmé sur une période de I2 heures est moins précise, mais elle risque d'être systématiquement inférieure à celle que les agneaux consomment réellement lorsqu'ils sont en liberté avec leur mère.

\section{4. - Fréquence des contrôles journaliers.}

Pour estimer la production laitière des brebis pendant l'allaitement, il faut effectuer un certain nombre de contrôles journaliers. Le problème de la détermination de la fréquence de ces contrôles nécessite la connaissance exacte de la production journalière des brebis au cours de toute la période d'allaitement, ce qu'il n'est pas possible d'envisager. Cependant, à partir des résultats obtenus dans le cadre de la traite des brebis, nous pouvons estimer les erreurs introduites dans le calcul de la lactation en espaçant l'intervalle entre contrôles journaliers.

Ces erreurs ont pour origine deux principales sources de variation :

- la variabilité de la production laitière d'un jour à l'autre indépendamment de l'évolution de la lactation ;

- les fluctuations de la production au cours d'une période de la lactation.

\section{$\mathrm{I}^{\mathrm{O}}$ Variabilité journalière.}

Pour les brebis soumises à la traite, le coefficient de variabilité de la production laitière journalière est de l'ordre de 7 à 9 p. IOo (RrCorDEAU-DFNaMUR).

Ce coefficient est exprimé d'après la moyenne des rapports :

$$
\frac{\text { Total lait jour }(\mathrm{J}+\mathrm{I})-\text { Total lait jour }(\mathrm{J})}{\text { Total lait jour }(\mathrm{J})} \times \text { Ioo. }
$$




\section{$2^{\circ}$ Espacement des contrôles journaliers.}

L'espacement des contrôles journaliers, pour des brebis soumises à la traite, introduit une certaine erreur dans le calcul de la lactation. Dans une autre étude (RICORDEAU) nous avons calculé l'écart entre production réelle et production calculée, en fonction de la périodicité des contrôles. Dans le cas d'un contrôle tous les 7 et I4 jours, les écartstypes des différences en valeur relative entre la production calculée et la production réelle sont respectivement de l'ordre de 2,0 p. Ioo et 2,6 p. IOO.

Si l'on admet, dans le cas d'allaitement, des résultats équivalents à ceux obtenus lors de la traite (ce que l'on a pu vérifier partiellement à partir de contrôles journaliers poursuivis pendant II jours consécutifs), 1a réalisation d'un contrôle hebdomadaire nous apparaît comme étant très acceptable.

\section{CONCLUSIONS \\ Méthode de référence.}

Pour mesurer la quantité de lait consommée par les agneaux, il est nécessaire d'effectuer les tétées toutes les 2 heures, au cours des premières semaines de lactation ; au-delà de 4 semaines, une tétée toutes les 3 heures est suffisante.

Il n'y a pas de différences significatives, du point de vue production laitière, entre 2 jours successifs de contrôle ou 2 demi-journées de contrôle. Nous proposons la méthode de référence suivante :

- I contrôle journalier par semaine,

- le premier contrôle journalier a lieu 4 à 5 jours après la misebas,

- chaque contrôle journalier comporte outre la pesée zéro effectuée très tôt le matin :

6 pesées à 2 heures d'intervalle

ou 4 pesées à 3 heures d'intervalle.

\section{SUMMARY}

After studying the natural suckling behaviour of lambs we have sought to determine a precise and practical method of estimating the milk production of ewes during the suckling period by weighing the lambs before and after suckling.

I. The suckling interval of 'lexel and Prealpes lambs is on an average about I I/2 hours; it is never more than 3 hours.

There is little difference in behaviour of suckling lambs whether they are single or twins.

The suckling frequency varies during the day and in relation to the weight of the lambs, but the most important factor is the age of the lambs. 
2. We recorded the milk production of $\mathrm{I}_{4}$ Prealpes ewes over two periods of 48 hours, after double weighings carried out every two hours and every three hours.

From the point of view of milk production there is no significant difference between two successive days'control or two half days'control.

The estimation of the milk production in 24 hours is valid when the weighings relate to a period of 12 .hours, with suckling intervals of 2 or 3 hours In both cases, the standard deviation of relative differences between real. production and estimated production is around 6 to $7 \mathrm{p}$. Ioo.

\section{RÉFÉRENCES BIBLIOGRAPHIQUES}

Barnicoat C. R., Logax A. G. et Grant A. T., I949. Milk secretion studies with New-Zealand Romney ewes. Part. I. J. Agr. Sci., 39, 44-55.

Bonsma F. N., I944. Milk production studies with sheep. Agr. Res. Inst. Série 5, Bul. 25I.

Burris M. J. et Baugus C. A., I955. Milk consumption and growth of suckling lambs. J. Anim. Sci., 14, I86-I9I.

Constantinescu O. et Gondos G., I958. Controlul productiei de lapte la oi in timpul alaptarii mieilor. Anal. Inst. Cerc. Zoot., 15, 459-470.

Davies H. L., I958. Milk yield of Australian Merino ewes and lamb growth under pastoral conditions. Proc. Aust. Soc. Anim. Prod., 2, I5-2I.

FUli.er J. G. et Kisinheinz F., Ig04. On the daily yield and composition of milk from ewes of various breeds. 2 Ist ann. Rep. Wisconsin Stat., 48-50 (in Exp. Stat. Record 16, 815, Ig05).

GUyer P. Q. et Dyer A. J., I954. Study of factors affecting sheep production. Missouri Agr. Exp. Stat. Res. Bull., $55^{8}$.

HugO W. J., I952. The influence of feeding on the milk production of the Merino ewes. Fmg in S. Afr., 2\%, 503.

HUnTER G. I., I956. The maternal influence on size in sheep. J. Agric. Sci., 48, 36-60.

LiERoy A.M., I936. Application des méthodes de contrôle laitier à la sélection des races ovines précoces. C. R. Acad. Agric., 22, 733-738.

Munro J., I956. Observations on the suckling behaviour of young lambs. Brit. J. Anim. Behav., 4, 34-36.

Munro J. et Ixisson R. H. E... Iy57. The effects of different suckling frequencies on the quantity of milk consumed by young lambs. J. Agric. Sci., 49, I69-I70.

NeIdig R. E. et IDdings E. J., IgIg. Quantity and composition of ewes' milk; its relation to the growth of lambs. J. Agric. Res., 17, 19-33.

OwEx J. B., I956. A study of the lactation and growth of Hill sheep in their native environment and under lowland conditions. J. Agric. Sci., 48, $387-4 \mathrm{I} 2$.

PIERCE A. W., I934. 'The yields and composition of the milk of the Merino ewe. Aust. J. Exper. Biol. Med. Sci., 12, 7-12.

RICORDEAU G. et DENAmUR R. (données non publiées).

SALMON-LEgagNeur E., r956. La mesure de la production laitière chez la truie. Ann. Zoot., 5 (2), 95-I IO.

Starke J.S., I953. Studies on the inheritance of milk production in sheep. S. Afr. J. Sci., 49, $245-254$.

Thomson W., I952-I953. Feeding the in lamb ewe. Scot. Agric., 32, I2O-I 24. 
Thomson W. et Thomson A. M., I953. Effect of diet on milk yield of the ewe and growth of her lamb. Brit. J. Nutr., \%, 263-274.

VERA Y VEGA A., I956. Comparacion de dos methodos de estimacion de la produccion lechera en la oveja durant el periodo de amamantamiento del cordero. Arch. Zootech., 5, 322-348.

WALIACE L. R., I948. Growth of lambs before and after birth in relation to the level of nutrition. Part. I. J. A gric. Sci., 38, 93-I53. 